\title{
Astrocitoma anaplásico do cerebelo: relato de caso
}

\author{
Márcia Raquel Tizziani', Alfonso Ramos de Castro Velasco², \\ Rodrigo Pedrosa Sobrido², António José Verdelho ${ }^{3}$ \\ Serviço de Neurocirurgia do Centro Hospitalar do Porto. Hospital Geral de Santo António, Porto, Portugal.
}

\section{RESUMO}

Astrocitomas de alto grau são tumores de apresentação rara na fossa posterior, especialmente na idade adulta. A sobrevida média descrita na literatura é de cerca de 14 a 32 meses. A apresentação clínica mais frequente é na forma de síndrome de hipertensão intracraniana. Tende a evoluir com disseminação leptomeníngea e também pode estender-se ao tronco cerebral e à medula. Apresentamos um caso de astrocitoma anaplásico (grau III) da fossa posterior numa mulher de 62 anos, o único observado num adulto no nosso hospital num período de 10 anos. O diagnóstico foi confirmado com biópsia estereotáxica da lesão. A apresentação clínica está de acordo com o descrito na literatura, contudo a sobrevida observada foi significativamente mais curta.

\section{PALAVRA-CHAVE}

Atrocitoma anaplásico cerebelar.

\section{ABSTRACT \\ KEYWORD \\ Anaplastic cerebellar astrocytoma.}

Anaplasic cerebellar astrocytoma: case report

High grade astrocytomas are rare tumors in posterior fossa, especially in adulthood. The median survival reported in literature is around 14 to 32 months. The most frequent clinical presentation is intracranial hypertension syndrome. It tends to present leptomeningeal dissemination and may also extend to the brainstem and spinal cord. We present a case of anaplastic astrocytoma (grade III) in the posterior fossa in a 62 year-old female, the only adult presentation of this tumor observed in our hospital over a period of 10 years. The diagnosis was confirmed by stereotactic biopsy of the lesion. The clinical presentation is consistent with that described in the literature, however the survival time was significantly shorter.

\section{Introdução}

Astrocitomas de alto grau são tumores de apresentação rara na fossa posterior em qualquer faixa etária, ${ }^{3-7}$ especialmente na idade adulta. Há poucos casos relatados na literatura e não há uma explicação para a raridade nessa localização. A sobrevida média descrita é de cerca de 14 a 32 meses. ${ }^{5,7,8}$ Parte dos casos relatados associa o desenvolvimento de gliomas malignos do cerebelo à exposição prévia a radioterapia para tratamento de outro tumor cerebral. ${ }^{12}$ Incidem mais no hemisfério cerebeloso direito por motivos desconhecidos. A apresentação clínica mais frequente é na forma de síndrome de hipertensão intracraniana ${ }^{4}$ Tendem a evoluir com disseminação leptomeníngea ${ }^{5,7}$ e também podem estender-se ao tronco cerebral e à medula. ${ }^{3,6,7}$ O tratamento cirúrgico é indicado nos casos em que a abordagem e a remoção tumoral não acrescentem déficits neurológicos ao paciente. Embora haja controvérsias quanto ao tratamento, geralmente são utilizadas radioterapia e quimioterapia paliativas. ${ }^{1,3,5,7,11,13}$

\section{Relato do caso}

Doente de 62 anos, sexo feminino, aposentada (trabalhava com contabilidade). O quadro clínico começou com vertigem, tinitus e cefaleia occipital de leve intensidade, com episódios esporádicos de vômitos,

1 Médica contratada do Serviço de Neurocirurgia do Centro Hospitalar do Porto - Hospital Geral de Santo António.

2 Médico interno complementar de Neurocirurgia do Centro Hospitalar do Porto - Hospital Geral de Santo António.

3 Médico-assistente hospitalar de Neurocirurgia do Instituto Português de Oncologia. 
evoluindo com desequilíbrio da marcha, com quedas para a direita. Quadro progressivo sem melhoria com medicação sintomática, evoluindo com incapacidade de marcha autônoma após um mês. Foi investigada com tomografia cerebral, que revelou lesão expansiva intra-axial da fossa posterior com deformação do IV ventrículo, sem sinais de hidrocefalia. Hipóteses diagnósticas de metástase cerebral, hemangioblastoma ou tumor da série glial. De antecedentes relevantes, tinha sido submetida a histerossalpingectomia e ooforectomia por lesão pré-cancerosa, cinco anos antes, sem necessidade de tratamentos complementares. Tinha história familiar de tumor cerebral (irmão).

Ao exame físico, apresentava bom estado geral, sem alterações cognitivas, ligeiro déficit auditivo à direita, ligeira dismetria à direita, marcha com desequilíbrio para a direita. Restante dos exames físico e neurológicos normais. Karnofsky de 80.

Investigada com ressonância magnética (RM) cerebral (Figura 1): lesão expansiva hemisférica cerebelosa direita, intraparenquimatosa, captante de contraste de forma heterogênea, com envolvimento do pedúnculo cerebeloso médio e do tronco cerebral e que aparentemente atinge o flóculo cerebeloso. Ausência de hidrocefalia. Hipótese de lesão metastática ou tumor da série glial.

Iniciou corticoterapia e foi internada por causa da hipertensão intracraniana.

Pela hipótese de metástase, procedeu-se ao rastreio de possível tumor primário:

- Tomografia computadorizada (TC) tóracoabdomino-pélvica: espessamento marcado da parede do cólon direito.

- Mamografia: sem evidência de lesão oncológica.

- Colonoscopia: pólipo do reto.

- Endoscopia digestiva alta: normal.

- Exame citopatológico do colo do útero: negativo para lesão intraepitelial ou neoplasia maligna.

Como a lesão invadia o pedúnculo cerebeloso e o tronco cerebral, foi proposta biópsia estereotáxica (Figura 2). Com a paciente sob sedação, em decúbito lateral esquerdo, utilizou-se o esterotaxímetro Leksell ${ }^{\circledR}$ com montagem anteroposterior do arco e ponto de entrada na fossa posterior, utilizando agulha de biópsia de Sedan com janela lateral. Procedimento sem intercorrências.
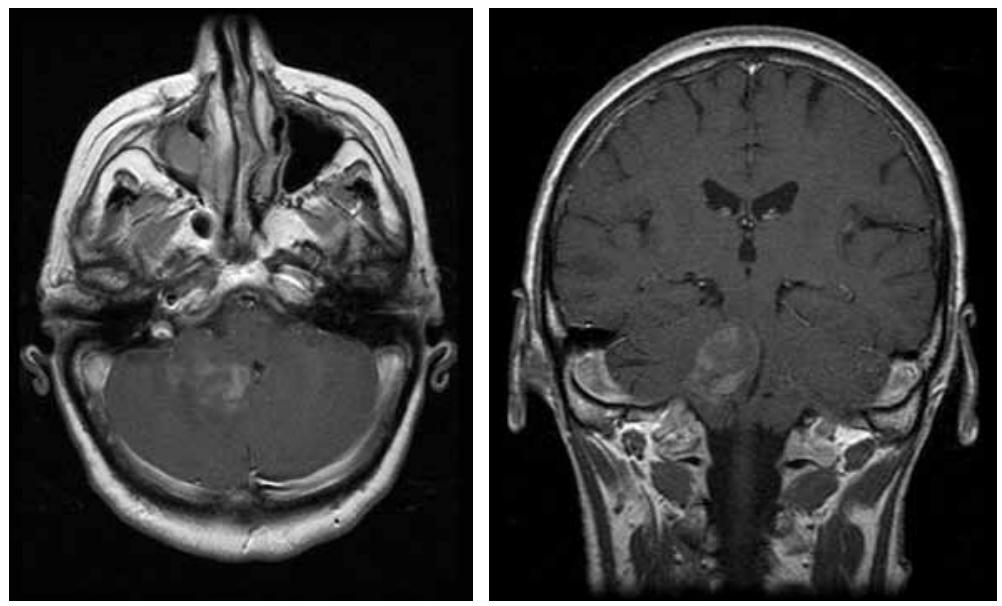

Figura 1-RM cerebral.

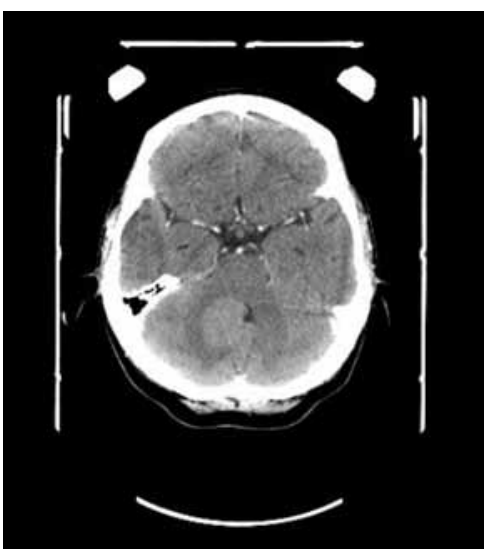

Figura 2 - Definição do alvo para biópsia estereotáxica, sendo escolhida área de maior captação de contraste. Exame extemporâneo: tumor da série glial.

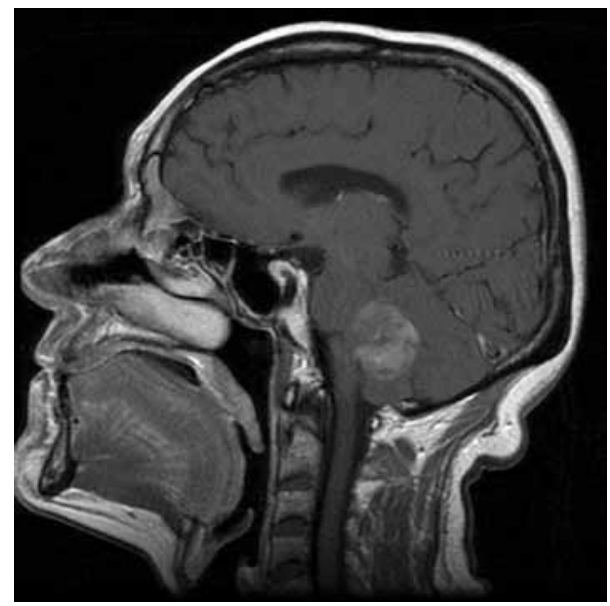


À data de alta, mantinha queixas de vertigens, referia hipoestesia no membro inferior direito e tinha discreta paresia desse membro. Em retirada gradual de corticoide, aguardando histologia definitiva para decisão terapêutica em consulta de grupo multidisciplinar.

$\mathrm{O}$ exame histológico definitivo mostrou tratar-se de tumor astrocitário (Figura 3) com numerosas fibras de Rosenthal, vasos com proliferação endotelial, anaplasia e índice de proliferação nuclear aumentado, sugerindo tratar-se de astrocitoma agressivo, embora não se observando mitoses nem necrose. Concluído ser astrocitoma grau III.
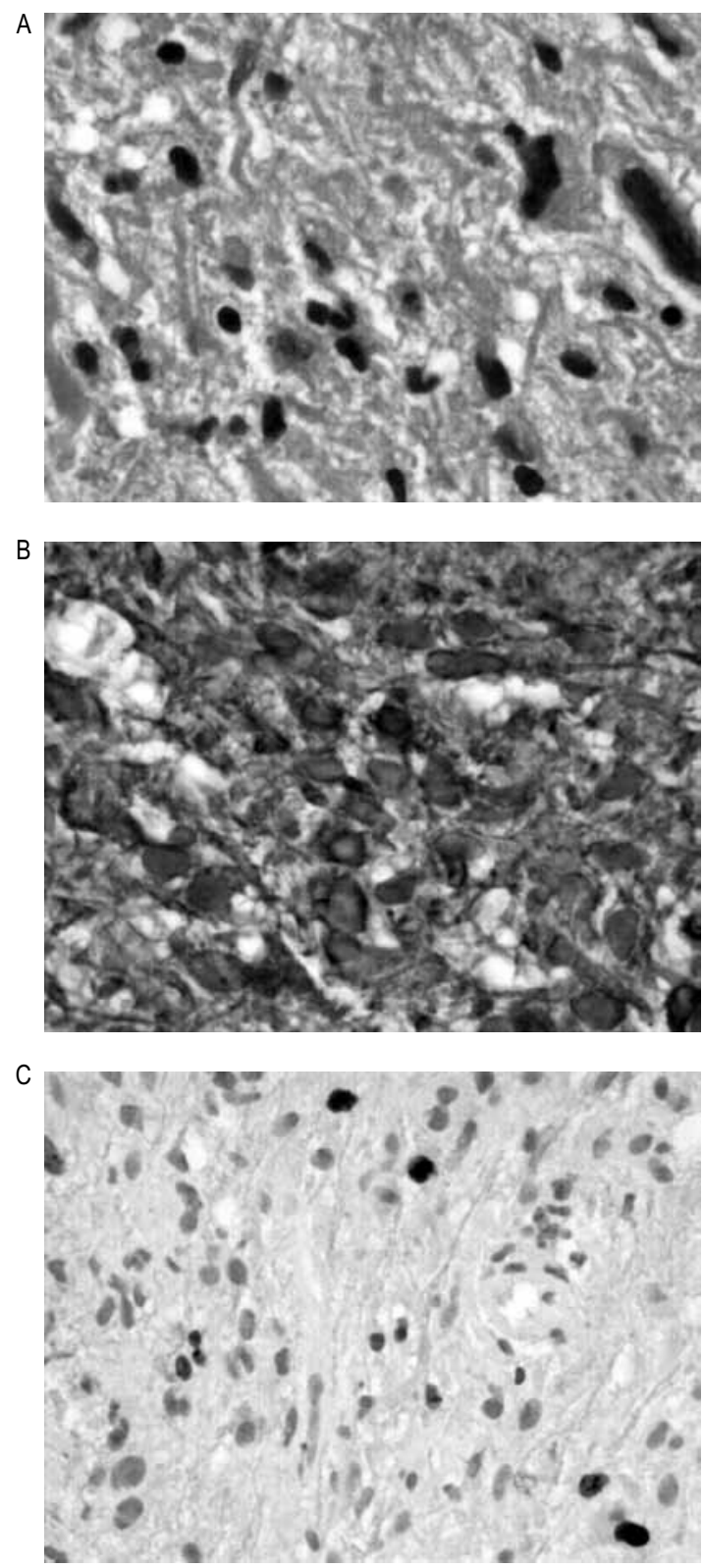

Figura 3 -Histologia. Em A, verifica-se anaplasia nuclear; em B (GFAP), grande parte das células mostra positividade; em C (KIG+), observa-se proliferação nuclear moderada.
Cerca de três a quatro semanas após a alta, evoluiu com hemiparesia direita, cefaleia, vômitos e disfagia, progressivamente mais prostrada e sem resposta verbal, desenvolveu paresia facial direita central e paresia do VI par craniano bilateral. TC (Figura 4) mostrou hidrocefalia.
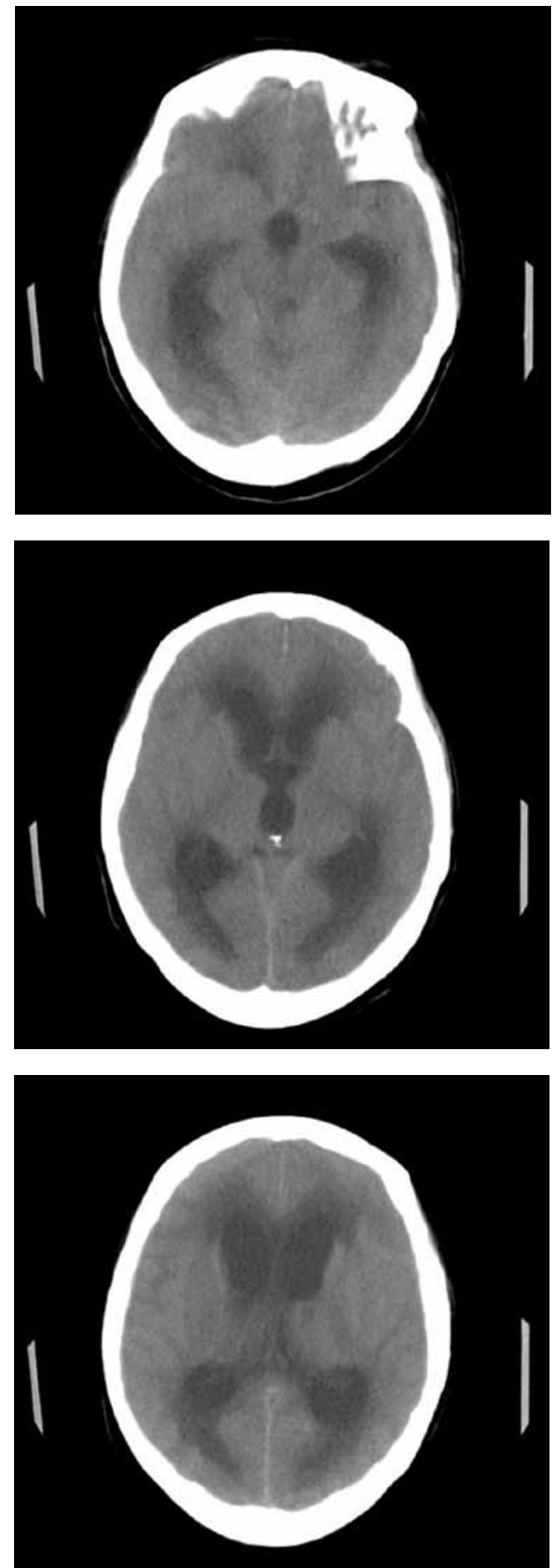

Figura 4 - TC cerebral mostrando apagamento do IV ventrículo com hidrocefalia ativa importante. 
Foi submetida à derivação ventrículo-peritoneal (DVP). Apresentou melhoria do estado de consciência no pós-operatório inicial, voltando a deteriorar três dias após. Confirmou-se com TC cerebral que a hidrocefalia estava tratada e analiticamente foram excluídas alterações metabólicas. Verificou-se óbito no sexto dia do pós-operatório da DVP.

\section{Discussão}

Dado o aspecto imagiológico da lesão, com caráter expansivo e heterogêneo, e atendendo à idade da doente, coloca-se a questão da amostragem da biópsia estereotáxica. Biopsiou-se uma área de maior captação de contraste, mas poderia estar subvalorizada a classificação na amostra por ter sido atingida uma área sem mitoses e/ou necrose, embora com proliferação nuclear.

A nossa doente evoluiu com alterações neurológicas compatíveis com atingimento da via piramidal e do tronco cerebral, que já era demonstrada imagiologicamente na RM inicial. A sobrevida da paciente foi menor do que a média relatada na literatura para astrocitomas anaplásicos do cerebelo. Antes do agravamento neurológico, a paciente não havia sido submetida a radioterapia ou quimioterapia complementar.

\section{Conclusão}

Apresentamos um caso raro de astrocitoma grau III da fossa posterior no adulto, o único observado no nosso hospital num período de 10 anos.

A apresentação clínica está de acordo com o descrito na literatura, contudo a sobrevida observada foi significativamente mais curta. Pensamos haver dois fatores importantes que condicionaram essa evolução: por um lado uma possível subvalorização do grau de malignidade histológica de acordo com a classificação da Organização Mundial da Saúde (OMS), o que pode estar relacionado com a amostragem da biópsia estereotáxica; por outro lado, a proximidade imagiológica ou sinais clínicos de invasão do tronco cerebral, que parecem ser determinantes para a evolução negativa do caso.

É questionável a utilidade de cirurgias de derivação de líquor nos doentes com astrocitomas malignos invadindo o tronco.

\section{Referências}

1. Bristot R, Santoro A, Raco A, Salvati M, Puzzilli F, Delfini $\mathrm{R}$, et al. Mallignant cerebellar astrocytomas: clínicopathological remarks on 10 cases. J Neurosurg Sci. 1999;43:271-6.

2. Chamberlain MC, Silver P, Levin VA. Poorly differentiated gliomas of the cerebellum: a study of 18 patients. Cancer. 1990;65:337-40.

3. Debono B, Derrey S, Rabehenina C, Proust F, Freger P, Laquerrière A. Primary diffuse multinodular leptomeningeal gliomatosis: case report and review of the literature. Surg Neurol. 2006;65:273-82.

4. Endo H, Kumabe T, Jokura H, Shirane R, Ariga H, Takai Y, et al. Leptomeningeal dissemination of cerebellar malignant astrocytomas. J Neurooncol. 2003;63:191-9.

5. Hanyu $\mathrm{H}$, Yoneda $\mathrm{Y}$, Katsunuma $\mathrm{H}$, Miki T, Miwa T. Wallemberg's syndrome caused by a brain tumor: a case report and literature review. Rinsho Shinkeigaku. 1990;30:324-6.

6. Kitaoka K, Ito T, Tashiro K, Abe H, Tsuru M, Miyasaka K. Vertebral angiography of cerebellar astrocytoma tumor stain, tumor circulation, CT and angiography in diagnosis. No Shinkei Geka (Tokyo). 1982;10:501-9.

7. Manabu K, Kazumi Y, Tetsu G. Cerebellar anaplastic astrocytoma mimicking cerebellar hemorrhage: case report and review of the literature. J Osaka Rosai Hosp. 2006;28:39-44.

8. Mattos JP, Marenco HA, Campos JM, Faria AV, Queiroz LS, Borges G, et al. Cerebellar glioblastoma multiforme in na adult. Arq Neuropsiquiatr. 2006;64:132-5.

9. Nakamizo A, Nishio $S$, Inamura $T$, Koga $H$, Yamabe $K$, Kuba $\mathrm{H}$, et al. Evolution of malignant cerebellar astrocytoma at the site of a treated medulloblastoma: report of 2 cases. Acta Neurochir (Wien). 2001;143:697-700.

10. Rizk T, Remond J, Alhayek G, Fournier D, Manei P, Mercier $\mathrm{P}$, et al. Malignant astrocytoma of the cerebellum. Apropos of 10 cases. Review of the literature. Neurochirurgie. 1994;40:121-6.

11. Tsunoda S, Sakaki T, Kubota T, Goda K, Nakamura $\mathrm{M}$, Hashimoto $\mathrm{H}$, et al. Anaplastic astrocytoma of an oncocytic type occurring in the cerebellar vermis in Pierre Robin syndrome: case report. Neurol Med Chir (Tokyo). 1992;32:911-4.

12. Viano JC, Herrera EJ, Suárez JC. Cerebellar astrocytomas: a 24-year experience. Childs Nerv Syst. 2001;17:607-10.

13. Wisoff JH, Boyett JM, Berger MS, Brant C, Li H, Yates AJ, et al. Current neurosurgical management and the impact of the extent of resection in the treatment of malignant gliomas of childhood: a report of the Children's Cancer Group trial no. CCG-945. J Neurosurg. 1998;89:52-9.

Endereço para correspondência

Márcia Raquel Tizziani

Av. Serpa Pinto, 404, casa C

4450-277 - Matosinhos, Portugal

E-mail:marciatizzi@hotmail.com 